\title{
Meta
}

Journal des traducteurs

Translators' Journal

\section{BAIGORRI JALÓN, J. (2004) : Interpreters at the United Nations : A History, Salamanca, Ediciones Universidad de Salamanca, $181 \mathrm{p}$.}

\section{Marjorie Agrifoglio}

Volume 50, numéro 3, août 2005

Le prisme de l'histoire

The History Lens

URI : https://id.erudit.org/iderudit/011621ar

DOI : https://doi.org/10.7202/011621ar

Aller au sommaire du numéro

Éditeur(s)

Les Presses de l'Université de Montréal

ISSN

0026-0452 (imprimé)

1492-1421 (numérique)

Découvrir la revue

Citer ce compte rendu

Agrifoglio, M. (2005). Compte rendu de [BAIGORRI JALÓN, J. (2004) : Interpreters at the United Nations : A History, Salamanca, Ediciones Universidad de

Salamanca, 181 p.] Meta, 50(3), 1067-1069. https://doi.org/10.7202/011621ar

Ce document est protégé par la loi sur le droit d'auteur. L'utilisation des services d'Érudit (y compris la reproduction) est assujettie à sa politique d'utilisation que vous pouvez consulter en ligne.

https://apropos.erudit.org/fr/usagers/politique-dutilisation/ 
était de mise par rapport aux difficultés, notamment la piètre qualité acoustique et la mauvaise insonorisation des cabines.

On retiendra de ce livre l'«interprétation personnelle» de l'histoire de l'interprétation de conférence. L'auteur a posé son regard d'interprète sur une grande variété de documents pour écrire un récit amène et riche en informations sur la naissance d'une profession récente qui gagne à être mieux connue.

Ce livre s'arrête là où commence un deuxième volume tout aussi intéressant sur les interprètes des Nations Unies.

Mayra S. PARra

Université de Montréal, Montréal, Canada

\section{RÉFÉRENCES}

BAigorri JaLón, J. (2000): La interpretación de conferencias: el nacimiento de una profesión. De París a Nuremberg, Granada, Editorial Comares.

Gaiba, F. (1998): The Origins of Simultaneous Interpretation. The Nuremberg Trial, Ottawa, Les Presses de l'Université d'Ottawa.

Baigorri Jalón, J. (2004): Interpreters at the United Nations: A History, Salamanca, Ediciones Universidad de Salamanca, $181 \mathrm{p}$.

Les Nations Unies incarnent un des rêves les plus sublimes de l'humanité, celui de la recherche d'un consensus entre toutes les nations de la planète. C'est dans ce forum multilingue que le rêve des pionniers de la simultanée est devenue réalité: cette modalité s'est consolidée comme la reine de l'art de l'interprétation de conférence. Ce deuxième ouvrage de Jesús Baigorri Jalón, historien, ancien interprète aux Nations Unies et aujourd'hui professeur d'interprétation à l'Université de Salamanca, est la suite du premier. Toujours minutieux et pondéré, l'auteur continue de retracer l'histoire de la profession d'interprète de conférence en nous menant, cette fois, jusqu'à nos jours.

Traduit d'un manuscrit espagnol (non publié) par Anne Barr et publié (en anglais!) à Salamanca, Interpreters of the United Nations: A History se veut une contribution à la biographie de la profession, axée sur celle des interprètes qui ont travaillé ou qui travaillent encore au sein de cette célèbre organisation internationale. Une histoire et non pas l'histoire, Baigorri tient à le souligner dès les premières pages. D'une part, cet "essai» ne vise pas à être un répertoire de tous les interprètes du passé des Nations Unies. D’autre part, comme dans toute histoire, le récit constitue une interprétation parmi d'autres, empreinte de la subjectivité de son auteur: "This book does not aim to be objective or neutral, among other reasons because history is never neutral» (p. 12). Un postulat précieux pour ceux qui s'aventurent dans la recherche historique en traductologie, d'autant plus qu'il est énoncé par un historien. Néanmoins, cette subjectivité n'exclut pas la rigueur, tel que l'illustrent les quatre chapitres de cet ouvrage clair et bien structuré, qui se fondent principalement sur des sources orales et des documents d'archives de l'ONU.

Le premier chapitre donne un bref aperçu des débuts du multilinguisme aux Nations Unies. Les lecteurs y apprendront que, contrairement à ce que l'on pourrait croire, l'idée d'imposer d'autres langues de travail que l'anglais ne ralliait pas tous les suffrages lorsque l'organisation a été mise sur pied. Par ailleurs, si les pays victorieux de la Deuxième Guerre mondiale n'avaient pas été ceux qu'on connaît, les interprètes des Nations Unies traduiraient probablement aujourd'hui vers des langues autres que les six langues officielles. Baigorri raconte que c'est grâce aux pressions politiques des délégations de l'Amérique latine, de la 
Chine, de la France et de l'Union soviétique que l'espagnol, le chinois, le français et le russe sont devenus des langues officielles. De cette décision, plus politique que communicative, découlera l'instauration de l'interprétation simultanée, qui venait d'être couronnée de succès à Nuremberg. Cette modalité fut officiellement adoptée le 30 octobre 1947. La consécutive n'est toutefois pas éliminée. Plus fiable et exacte - aux dires des délégués - que la simultanée lorsqu'il s'agissait de traduire des discours lus, la consécutive vers l'anglais et vers le français continuera d'être utilisée au sein du Conseil de sécurité pendant les premières années de l'organisation (même si les discours sont interprétés en simultanée aussi). Au fil du temps, les délégués du Conseil de sécurité se contenteront de la simultanée et la consécutive perdra définitivement sa place aux Nations Unies. Pour Baigorri, l'existence de services de traduction et d'interprétation semble relever davantage des principes couchés dans les règlements que d'un vrai besoin de communication, surtout à l'ère de la mondialisation.

Le deuxième chapitre est consacré aux interprètes de la première période définie par l'auteur, celle qui s'étend de 1945 à 1960. Cette période est marquée par un des épisodes les plus intéressants de l'histoire de l'interprétation contemporaine: la guerre entre les «vétérans» de la consécutive, les interprètes chevronnés de la Société des Nations, et les nouveaux «téléphonistes», les pionniers de la simultanée, issus de Nuremberg. À la tête de chaque camp figure un des grands noms de la profession: Jean Herbert et Léon Dostert, respectivement. Craignant la perte de leurs privilèges, les «vétérans» ont mené une campagne auprès des délégués pour les convaincre de l'intérêt de la consécutive au cours des débats, celle-ci accordant du temps supplémentaire pour se préparer aux réponses. Pour souligner la valeur formative de l'histoire et la pertinence des regards vers le passé, Baigorri compare ce rejet de la simultanée à la crainte que suscite de nos jours la nouvelle technologie de la simultanée à distance chez plusieurs interprètes.

La guerre sera enfin remportée par Dostert qui a su s'appuyer sur les nouvelles recrues pour imposer sa thèse aux délégués. Et du sang nouveau, il en fallait, bien entendu! Pendant cette période, le seul véritable critère de recrutement était la connaissance de plusieurs langues. Lors de la sélection, les candidats entraient directement en cabine et cette performance, la première de leur vie, déterminait leur embauche. Déracinés, exilés ou enfants de la noblesse russe, les premiers interprètes étaient des bilingues «naturels» et ne cherchaient pas à faire carrière en interprétation. Ils ont hérité de l'image de "prodige» des vétérans de la Société des Nations et, au lieu de la démystifier, ils l'ont perpétuée.

La génération de transition (1960-1980), objet du troisième chapitre, a été témoin de plusieurs changements survenus dans l'activité d'interprétation, dont le plus important est sans aucun doute celui de la professionnalisation. Contrairement aux interprètes de la première génération, ceux de la deuxième n'arrivent pas à la profession par hasard, ni à la suite de cataclysmes mondiaux. Diplômés universitaires dans des domaines tels que l'histoire, l'enseignement, les langues ou la traduction, ils réussissent l'examen d'admission, encore très subjectif et aléatoire, et deviennent des interprètes «onusiens».

C'est cette génération de transition qui a fait la grève de 1974 pour l'amélioration des conditions de travail et pour la réduction de la durée des prestations. Les revendications obtenues par les interprètes à l'issue de cette grève, dirigée par la cabine française, figurent aujourd'hui dans les règles de l'Association internationale d'interprètes de conférence (AIIC). Selon Baigorri, la grève rend manifeste la dépersonnalisation de la profession : l'interprète cesse d'être un prodige pour devenir anonyme. Derrière la grève, se dissimule la frustration des interprètes qui découvrent leurs limites.

Le quatrième et dernier chapitre concerne les interprètes actuels de l'ONU. Mettant en garde contre la difficulté d'évaluer le passé récent et contre l'impossibilité d'avoir accès à de nombreux documents d'archives, Baigorri insiste sur les deux aspects principaux de cette période: la féminisation de la profession et le poids des diplômés des écoles d'interprétation réputées. Une des raisons de l'augmentation du nombre de femmes, qui est passé de $21 \%$ en 
1950 à $69 \%$ en 1980, est la professionnalisation du métier. L'interprétation est devenue une profession à part entière et ne sert plus de tremplin vers des postes aux Nations Unies comme c'était le cas dans les années 1950. À l'instar des autres professions langagières, le métier d'interprète attire plus de femmes que d'hommes dans notre société occidentale. D'autre part, à l'heure actuelle, plus de la moitié des interprètes onusiens $(65 \%)$ détiennent un diplôme en interprétation. Ils ont un profil moins exotique que les exilés de la première génération et emploient un vocabulaire beaucoup plus technique et moins littéraire.

Les dernières pages du chapitre décrivent, avec une dose légère de sarcasme, les conditions actuelles de travail des interprètes aux Nations Unies. En effet, l'auteur brosse un tableau guère encourageant: réunions de plus en plus techniques, discours lus à une vitesse effroyable et parsemés de chiffres, et de "longues listes d'endroits éloignés qui figurent rarement sur les cartes et de leurs habitants dont le nom est seulement connu de leurs voisins les plus proches» (p. 153), argot onusien, omniprésence de l'anglais, qualité sous-optimale du son dans quelques cabines... Les interprètes professionnels se reconnaitront sans doute dans ces lignes et sauront gré à Baigorri d'avoir exposé les difficultés du métier. Les étudiants et les nouveaux diplômés, quant à eux, trouveront ces passages fascinants et révélateurs, bref, la vision d'un véritable «insider».

Enfin, en guise de synthèse dans une conclusion quelque peu répétitive, l'auteur reprend les lignes principales de son histoire de l'interprétation aux Nations Unies: l'évolution du profil sociologique des interprètes, l'évolution du processus de recrutement, le passage de la consécutive à la simultanée et la professionnalisation; le tout suivi de quelques commentaires sur l'avenir de la profession.

Les deux ouvrages de Baigorri témoignent d'un intérêt grandissant pour la recherche historique en traductologie. Les chercheurs qui s'engagent sur cette voie y trouveront un modèle à suivre. Outre la consultation de nombreuses sources orales originales, le mérite de Interpreters at the United Nations: A History réside dans l'emploi d'une méthodologie analytique-synthétique (López Alcalá 2001). Celle-ci se traduit par un récit qui présente les faits de façon rigoureuse, après en avoir confirmé la véracité dans plusieurs sources, et qui les met toujours en rapport avec les thèmes privilégiés par l'historien, dont le mythe de l'interprète prodige et le progrès technologique. En d'autres termes, ce livre comme le premier n'est pas une compilation érudite de dates et d'événements qu'on fait défiler en ordre chronologique sans autre perspective que le mouvement temporel (comme dans Roland 1999 et Gaiba 1998). La recommandation «Donnez un objectif à la recherche historique» qui a surgi de la table ronde sur la méthodologie de la recherche en histoire lors du XVII ${ }^{\mathrm{e}}$ Congrès de l'ACT tenu à Winnipeg en 2004 se voit appliquée dans cet ouvrage avec tous les honneurs. Grâce à Jesús Baigorri Jalón, la recherche historique en interprétation a franchi un pas important.

Marjorie Agrifoglio

Université de Montréal, Montréal, Canada

\section{RÉFÉRENCES}

López Alcalá, S. (2001) : La historia, la traducción y el control del pasado, Madrid, Universidad Pontificia Comillas.

Roland, R. (1999) : Interpreters as Diplomats, Ottawa, University of Ottawa Press. 